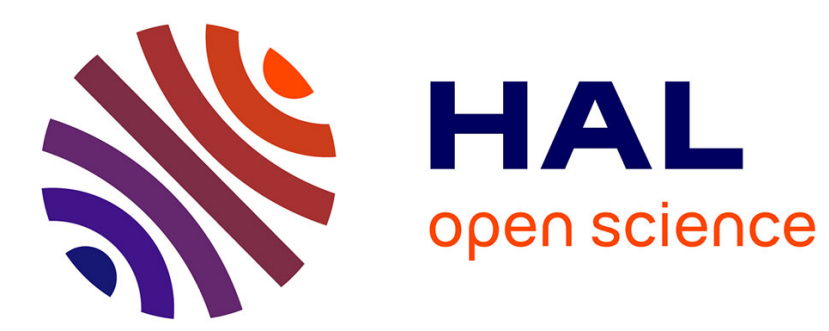

\title{
On the computation of the maximum capacity of TDMA-CDMA/TDD systems
}

\author{
Jad Nasreddine, Xavier Lagrange
}

\section{To cite this version:}

Jad Nasreddine, Xavier Lagrange. On the computation of the maximum capacity of TDMACDMA/TDD systems. PIMRC'06: 17th IEEE international symposium on personal, indoor and mobile radio, Sep 2006, Helsinki, Finland. 10.1109/PIMRC.2006.254199 . hal-02166926

\section{HAL Id: hal-02166926 \\ https://hal.science/hal-02166926}

Submitted on 27 Jun 2019

HAL is a multi-disciplinary open access archive for the deposit and dissemination of scientific research documents, whether they are published or not. The documents may come from teaching and research institutions in France or abroad, or from public or private research centers.
L'archive ouverte pluridisciplinaire HAL, est destinée au dépôt et à la diffusion de documents scientifiques de niveau recherche, publiés ou non, émanant des établissements d'enseignement et de recherche français ou étrangers, des laboratoires publics ou privés. 


\section{ON THE COMPUTATION OF THE MAXIMUM CAPACITY OF TDMA-CDMA/TDD SYSTEMS}

\author{
Jad Nasreddine \\ TSC Dpt., Universitat Politecnica de Catalunya \\ c/ Jordi Girona, 1-3, Campus Nord D4 \\ 08034 Barcelona, Spain \\ jnassred@tsc.upc.edu
}

\author{
Xavier Lagrange \\ RSM Dpt., GET/ENST de Bretagne \\ 2 rue de la châtaigneraie, CS 17607 \\ 35576 Cesson Sévigné Cedex, France \\ xavier.lagrange@enst-bretagne.fr
}

\section{ABSTRACT}

The combination of the TDD mode and the TDMA technique in WCDMA systems engenders a new degree of flexibility that can increase system capacity and QoS levels of active channels if efficient slot allocation techniques are used. These techniques allow the system to control the allocation of slots to users, and thus lead to relevant reduction in interference levels. In order to study slot allocation performance, it is important to define a reference performance that gives adequate approximation of system maximum capacity. In this paper, we propose a novel formulation to the computation of system maximum capacity using a set of combinatorial optimization problems. Furthermore, we propose meta-heuristic algorithms that give satisfactory approximations for each combinatorial problem.

\section{INTRODUCTION}

The exciting pace of mobile system evolution has characterized the last few years. Specifically, there is a substantial interest to merge packet-switched oriented services prevalent on Internet and mobile telecommunications systems to fulfill the vision of: "anyone, anytime and anywhere" [1]. In other words, next generation mobile systems are intended to provide the same services now available in the fixed network, such as asymmetric services with high data rates. These emerged services arouse new challenges for cellular system designer due to the complex and unstable nature of the radio interface. Thereby, the development of efficient Radio Resource Management (RRM) procedures is paramount for next generation mobile systems. The Code Division Multiple Access (CDMA) technique with a combination of Time Division Duplex (TDD) mode and Time Division Multiple Access (TDMA) technique introduce an interesting degree of flexibility to RRM potential. Namely, slot allocation techniques can be very advantageous due to their ability in avoiding high interference patterns. Nevertheless, the performance of these techniques are limited and this limitation should be specified. This can be done by computing the maximum capacity of TD-CDMA/TDD systems. This problem has been treated in the literature using different approaches such as in $[2,3]$. In this contribution, we propose a new approach based on decomposing the problem into a set of combinatorial optimization problems. Then, we propose a mechanism that combines optimum power control [4] and slot allocation techniques to solve each of the optimization problems. Optimum power control is used to achieve CIR-balancing [5] in each slot while the slot allocation algorithm exploits the flexibility of TDMA/TDD combination. Due to the fast convergence of the proposed approach, it can be applied to systems incorporating any number of cells or slots.

The proposed slot allocation technique is based on the principles of simulated annealing [6] which is a general metaheuristic technique dedicated to find a good solution to difficult optimization problems by avoiding local minima. The fundamental idea of simulated annealing is to allow moves that lead to worse quality than the current solution in order to escape from local minima. In this paper, we only consider downlink slots with the possibility to extend the proposed model to uplink and crossed slots using the power control model of [4].

The rest of the paper is organized as follows. In section II, the studied system is described. In section III, the optimization problem is formulated as a set of combinatorial problems and the CIR-balancing power control mechanism is described. Section IV is devoted to the re-allocation meta-heuristic algorithm. Before concluding, we present simulation results in section V.

\section{SYSTEM DESCRIPTION}

In the studied system, $N$ mobiles are uniformly distributed across $M$ cells with $N / M$ mobiles in each cell. Moreover, the system allocates $T_{d}$ slots for downlink. We assume that each mobile is assigned to one time slot in the TDMA frame.

Let $\Omega$ be the set of all active mobiles in the system. By convention, indices $i$ and $k$ always refer to mobiles while indices $j$ and $l$ refer to base stations. Furthermore, index $j$ refers to the base station that serves mobile $i$ and $S_{j}$ is the set of all mobiles communicating with base station $j$. The set $S_{j}$ is divided into $T_{d}$ subsets $S_{j}^{(n)}$, where $S_{j}^{(n)}$ is the set of mobiles connected to base station $j$ during slot $n$ and has cardinality $N_{j, n}$. The QoS of a mobile $i$ in a cell $j$ during slot $n$ is represented by the Carrier to Interference Ratio (CIR) and it is denoted by $\Gamma_{i, j, n}$. The transmitted power of a base station is upper-bounded by $P_{\max }$ and mobiles are supposed to be served by the best received base station. CIR value $\gamma$ is called achievable hereafter if all mobiles in the system can be served with a CIR equal or higher than $\gamma$. In order to evaluate the proposed approach, we consider the TDD mode of UMTS [7]. Hence, each slot can be allocated simultaneously to 16 codes at maximum in one cell.

\section{PROBLEM FORMULATION}

We assume that all mobiles have the same CIR threshold $\gamma_{t h}$ with the possibility of extending the proposed model to a more general case. Our objective is to develop an analytical model that find an approximation of TD-CDMA/TDD system maxi- 
mum capacity. Specifically, we intend to compute the maximum number of codes that can be supported under CIR and power constraints. The main problem is defined by the following General Allocation Optimization (GAO) problem:

$$
\left[\begin{array}{lll}
\max & N & \\
\text { subject to } & & \forall j, n \text { and } \forall i \in S_{j}^{(n)} \\
& \Gamma_{i, j, n} \geq \gamma_{\mathrm{th}} & \\
& \sum_{k \in S_{j}^{(n)}} P_{\mathrm{b}, k, n} \leq P_{\max } & \forall j, n \text { and } \forall i \in S_{j}^{(n)} \\
& N_{j, n} \leq 16 &
\end{array}\right.
$$

where $P_{\mathrm{b}, k, n}$ is the power transmitted to mobile $k$. This problem is a combinatorial problem with non-linear constraints. Thus, the research of the optimal solution is a combination of two NP-complete problems (i.e. mixed-integer programs [8] and non-linear programs [9]). Thus, it is an NP-hard problem [10] and the computational time could be unacceptable especially for high number of cells, slots and mobiles. Hence, we propose an indirect methodology to compute the maximum capacity by decomposing the global problem into a set of combinatorial optimization problems with non-linear constraints. In each intermediate combinatorial problem (called herein intermediate-problem), the number of mobiles is fixed and the maximum achievable CIR is computed. The number of mobiles is increased gradually by $M$ (i.e. one new mobile for each cell) until the maximum achievable CIR is less than $\gamma_{t h}$ (i.e. the system reaches its maximum capacity). This can be justified by the fact that we consider uniform mobile distributions. We start from $N=T_{d}$, which is certainly a feasible solution. In this case, each slot is allocated to only one mobile in the whole system. Thus, the only limiting factor is the thermal noise which should not prevent any mobile from reaching $\gamma_{t h}$ in a well dimensioned system.

\section{A. Optimum Power control}

The CIR $\Gamma_{i, j, n}$ can be written as:

$$
\Gamma_{i, j, n}=\frac{G_{i, j} P_{\mathrm{b}, i, n}}{I_{\text {intra }, i, n}+I_{\text {inter }, i, n}+N_{0}}
$$

where $G_{i, j}$ is the path gain between mobile $i$ and its server $j$, $N_{0}$ is background noise power, $I_{\text {intra }, i, n}$ is the intra-cell interference received by mobile $i$ from its server and $I_{\text {inter }, i, n}$ is the inter-cell interference received by mobile $i$ from neighboring base stations. $I_{\mathrm{intra}, i, n}$ and $I_{\mathrm{inter}, i, n}$ are given by [4]:

$$
\begin{aligned}
I_{\text {intra }, i, n} & =\beta_{\mathrm{d}} G_{i, j} \sum_{k \in S_{j}^{(n)}-\{i\}} P_{\mathrm{b}, k, n} \\
& =\beta_{\mathrm{d}}\left(1-\alpha_{i, n}\right) G_{i, j} \mathcal{P}_{\mathrm{T}, j, n} \\
I_{\text {inter }, i, n} & =\sum_{l \neq j} G_{i, l}\left(\sum_{k \in S_{l}^{(n)}} P_{\mathrm{b}, k, n}\right) \\
& =\sum_{l \neq j} G_{i, l} \mathcal{P}_{\mathrm{T}, l, n}
\end{aligned}
$$

where $\mathcal{P}_{\mathrm{T}, j, n}$ is the total power transmitted by base station $j$ during slot $n, \beta_{\mathrm{d}}$ is the intra-cell interference residual factor obtained by using joint detection technique [11] and $\alpha_{i, n}$ is the portion of $\mathcal{P}_{\mathrm{T}, j, n}$ dedicated to mobile $i$ during slot $n$.

The objective of the optimum power control procedure is to achieve CIR-balancing by maximizing the minimum CIR over all mobiles during a slot [12]. The obtained maximum value is called hereafter $\gamma_{n}$. Without loss of accuracy, we use the simplified model proposed in [4] to accelerate the computation. In this model, the optimum power control procedure is applied to the total power transmitted by base stations instead of the specific power of mobiles. Using this model, the optimum power control problem during slot $n$ can be written as:

$$
\frac{1+\gamma_{n}}{\gamma_{n}} \mathbf{P}_{n}=\mathbf{Z}^{(n)} \mathbf{P}_{n}+\mathbf{N}^{(n)}
$$

where $\mathbf{P}_{n}=\left[\mathcal{P}_{T, j, n}\right]$ is the $M \times 1$ vector of base station total transmitted powers, $\mathbf{N}^{(n)}$ is the $M \times 1$ vector of the normalized background noise with elements: $n_{j}^{(n)}=N_{0} \sum_{i \in S_{j}^{(n)}} \frac{1}{G_{i, j}}$ and $\mathbf{Z}^{(n)}$ is an $M \times M$ non negative matrix with elements:

$$
\mathcal{Z}_{j, l, n}= \begin{cases}1+\beta_{\mathrm{d}}\left(N_{j, n}-1\right) & \text { if } j=l \\ \sum_{i \in S_{j}^{(n)}} \frac{G_{i, l}}{G_{i, j}} & \text { Otherwise }\end{cases}
$$

The maximum achievable CIR can be obtained using the method proposed in [5]. In this method, a branch-and-bound scheme is used to find the highest $\gamma_{n}$ with the following constraint:

$$
P_{\max }=\max _{1 \leq r \leq N}\left[\left(\frac{1+\gamma_{n}}{\gamma_{n}} I_{N}-\mathbf{Z}^{(n)}\right)^{-1} \mathbf{N}^{(n)}\right]
$$

where $I_{N}$ is the $N \times N$ identity matrix.

Matrix $\left(\frac{1+\gamma_{n}}{\gamma_{n}} I_{N}-\mathbf{Z}^{(n)}\right)$ is non singular for all $\gamma_{n}$ smaller than the spectral radius $\rho\left(\mathbf{Z}^{(n)}\right)$, which is the eigenvalue of matrix $\mathbf{Z}^{(n)}$ with the highest modulus [13]. Therefore, the investigated values of $\gamma_{n}$ are between 0 and $\rho\left(\mathbf{Z}^{(n)}\right)$.

\section{B. New Formulation of the Optimization Problem}

In order to solve the GAO problem, we introduce the following proposition.

Proposition 1 Mobile allocations that maximize the minimum power-balanced CIR over all slots are the allocations that maximize the minimum CIR over all mobiles.

Proof. Let $\gamma_{n}^{(a)}$ be the power-balanced CIR computed during slot $n$ of allocation $\mathcal{A}$ and $\gamma_{\text {min }}^{(a)}$ be the minimum of $\gamma_{n}^{(a)}$ over all slots: $\gamma_{\min }^{(a)}=\min _{n} \gamma_{n}^{(a)}$. Furthermore, we denote by $\mathcal{S}$ one of the allocations that maximizes $\gamma_{\min }^{(a)}$ over all allocations. Using allocation $\mathcal{S}$, we obtain the maximum of $\gamma_{\min }^{(a)}$, denoted by $\gamma_{\min }^{(s)}: \gamma_{\min }^{(s)}=\max _{\mathcal{A}} \gamma_{\min }^{(a)}$. We assume that there exists another allocation $\mathcal{O}$ that maximizes the minimum CIR of all mobiles: $\mathcal{O}=\arg \max _{\mathcal{A}}\left[\min _{i \in \Omega}\left(\Gamma_{i, j, n}^{(a)}\right)\right]$ and the maximized minimum CIR is given by: $\Gamma_{\min }^{(o)}=\max _{\mathcal{A}}\left[\min _{i \in \Omega}\left(\Gamma_{i, j, n}^{(a)}\right)\right]$. This allocation is obtained by comparing the minimum CIR computed without the CIR-balancing procedure over all allocations. Let $\Gamma_{\min , n}^{(o)}$ be the minimum of $\Gamma_{i, j, n}^{(o)}$ during slot $n$. Thereby, we can write: $\Gamma_{\min }^{(o)}=\min _{n} \Gamma_{\min , n}^{(o)}$. As it was shown in [14], maximizing 
the minimum CIR during a slot corresponds to CIR-balancing. Thus, the minimum CIR in each slot of allocation $\mathcal{O}$ can be maximized by using the CIR-balancing procedure:

$$
\Gamma_{\min , n}^{(o)} \leq \gamma_{n}^{(o)} \quad \forall n \in\left\{1, \cdots, T_{d}\right\}
$$

Hence:

$$
\Gamma_{\min }^{(o)}=\min _{n \in\left\{1, \cdots, T_{d}\right\}} \Gamma_{\min , n}^{(o)} \leq \min _{n \in\left\{1, \cdots, T_{d}\right\}} \gamma_{n}^{(o)}=\gamma_{\min }^{(o)}
$$

However, $\gamma_{\min }^{(o)} \leq \gamma_{\min }^{(s)}$ (by definition of $\gamma_{\min }^{(s)}$ ) and therefore, $\gamma_{\min }^{(s)}$ is the highest achievable CIR.

In the problem definition, we had mentioned that at each intermediate-problem we seek to compute the maximum achievable CIR. Namely, we search for the allocation that maximizes the minimum CIR of all mobiles (i.e. optimal allocation). In the global problem, we want to compute the maximum number of mobiles that can be satisfied (i.e. served with a CIR higher than $\gamma_{t h}$ ). Therefore, the network must be able to satisfy the mobile with the lowest CIR $\gamma_{\text {min }}$, which is called performance-index. In other words, $\gamma_{\min }$ must be higher than $\gamma_{t h}$. Hence, we try to maximize this minimum in each intermediate-problem. We emphasize here that $\gamma_{\min }$ can be obtained in several allocations and that we are interested in any of these allocations. In fact, we only aim to compute $\gamma_{\min }$ and not to resolve a fairness problem. Nevertheless, this can be done by the means of a lexicographical optimization tool using our model (e.g. when $\gamma_{\min }$ is found, the corresponding slot is eliminated from the system and the algorithm is performed again to the remaining slots, etc.). Herein, we can rewrite our objective for each intermediate problem as:

\section{Find an allocation that maximizes the performance-index.}

Each intermediate-problem can be defined by the following Slot Allocation Optimization (SAO) problem:

$$
\left[\begin{array}{ll}
\max _{a} & \min _{i \in \Omega}\left(\max _{\mathbf{P}_{\ell} \leq P_{\max }} \Gamma_{i, j, n}\right) \\
\begin{array}{c}
\text { subject to } \\
\frac{1+\Gamma_{i, j, n}}{\Gamma_{i, j, n}} P_{\mathrm{b}, i, n} \geq \quad \\
\end{array} & \beta_{\mathrm{d}} \sum_{k \in S_{j}^{(n)}} P_{\mathrm{b}, k, n} \\
& +\sum_{l \neq j} \frac{G_{i, l}}{G_{i, l}}\left(\sum_{k \in S_{l}^{(n)}} P_{\mathrm{b}, k, n}\right) \\
& +\frac{N_{0}}{G_{i, l}} \forall j, n \text { and } i \in S_{j}^{(n)} \\
\sum_{k \in S_{j}^{(n)}} P_{\mathrm{b}, k, n} \leq & P_{\max } \\
N_{j, n} \leq & 16
\end{array}\right.
$$

The SAO problem is a combinatorial problem with $N$ nonlinear constraints, which is known to be an NP-hard problem [10]. Using proposition 1, the global optimization problem can be divided into two maximization problems to reduce its complexity: the first maximization computes the maximum achievable CIR during a slot using the simplified optimum power control, while the second find the allocation that maximizes the performance-index. The direct solution to find the optimal allocation is to study all possible allocations (i.e. brute force search). However, the number of these allocations increases exponentially with the number of mobiles. To give an idea on the magnitude of the number of all possible allocations, we consider a system with 490 mobiles uniformly distributed to 7 cells and 7 slots. The number of possible allocations computed using the derived equation in [15] is around $2 \times 10^{174}$. We must also add CIR-balancing power control complexity (i.e. first maximization), which is fortunately reduced by the simplified model [4]. The computation time needed to investigate all allocations, ranges from several days to several months depending on the complexity of tested algorithms and the computing power. Hence, we propose meta-heuristic algorithms that converge to allocations with high performance-indices.

\section{Channel Re-Allocation Algorithm}

In this section, we propose the Re-Allocation Meta-heuristic Algorithm (RAMA) to solve the SAO problem. RAMA uses the principle of simulated annealing to maximize the performance-index by reallocating new slots to active mobiles.

The power-balanced CIR is sensitive to the interaction between mobiles that share the same slot and not only to the characteristics of each mobile independently. Thus, a normal search can converge toward a local optimum; consider an allocation where the performance-index is higher than all other performance-indices in neighborhood allocations by only changing the allocated slot to one mobile. This allocation is considered as the optimum allocation if it is forbidden to move toward a solution with lower performance-index than the actual index. However, there is a possibility to reach allocations by reallocating more than one mobile and obtain higher performance-indices. In order to avoid these local optima we have used simulated annealing. In the following, we define some parameters and functions in order to introduce the different iterations of RAMA.

Solution space $\mathcal{X}$ involves all global mobile allocations $\mathcal{A}$ that verify the following two constraints: All mobiles are associated to slots and the number of simultaneous active mobiles during the same slot in each cell is less than 16 . The cost function $C(\mathcal{A})$ of allocation $\mathcal{A}$ is defined by: $C(\mathcal{A})=$ $-10 \log _{10}\left[\gamma_{\mathrm{min}}^{(a)}\right]$ (i.e. the function to minimize). The neighborhood structure $\mathcal{N}_{e}(\mathcal{A})$ of allocation $\mathcal{A}$ is built by changing the allocated slot of only one mobile. The transit solution involves two parameters: the mobile that must be reallocated and the new slot that will be allocated to the chosen mobile.

Two strategies may be used to choose the mobile. In both strategies, the chosen mobile must be initially active during the worst slot $w s$ defined by:

$$
w s=\arg \min _{n} \gamma_{n}^{(a)}
$$

Moreover, the mobile is chosen from mobiles served by base station $w b$ transmitting with the highest power during slot $w s$. We choose this base station, because it is the base station that imposes the CIR constraint in a noisy system. Thereafter, a mobile is chosen using one of two strategies: Worst Mobile Move (WMM) strategy and Best Mobile Move (BMM) strategy. In the WMM strategy, we select mobile $i$ with the highest sum of 


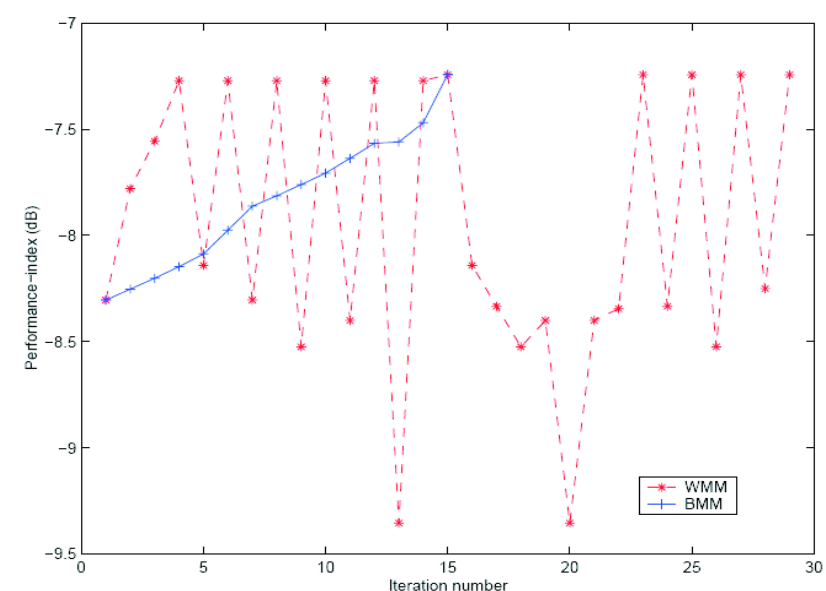

Figure 1: A convergence example of the RAMA when using either WMM or BMM strategies

normalized pathgains $z_{i}$. The normalized pathgain of a mobile toward a neighboring base station is the ratio of the pathgain between the mobile and the latter base station, and the pathgain between the mobile and its server. The chosen mobile is therefore, the mobile that experiences the highest inter-cell interference power when all base stations are transmitting with the same power. In the BMM strategy however, we select mobile $i$ with the lowest sum of $z_{i}$ (i.e. the mobile that experiences the lowest inter-cell interference power).

The WMM strategy allows fast convergence, while the BMM strategy guarantees stability in the system (i.e. the minimum CIR does not fall to low values) as depicted in figure 1. In this figure, we take a sample of geographical mobile distribution in a system with seven cells, seven slots and five mobiles/cell/slots. We can see that the WMM strategy leads to a degradation in the QoS level, even if it allows a faster convergence. This is due to the fact that the worst mobile could have high impact on the new slot. On the contrary, the BMM strategy converges more slowly but with an increasing QoS level. We tested several mobile distributions and noticed the same behavior in almost all cases. This result could be used to develop a practical slot allocation algorithm.

The new slot is selected from the solution space and must have the highest achievable CIR. If the transition is not accepted, another slot is selected with the same constraint until no slots are left or the transition is accepted. If all slots are investigated without success, the chosen mobile is neglected and a new mobile is chosen from the same cell using one of the two strategies, until all mobiles of this cell are tested without success. All other cells are then tested, starting by the cell with the second highest transmitted power.

The transition probability from allocation $\mathcal{A}$ to allocation $\mathcal{B}$ is the probability of accepting the new allocation $\mathcal{B}$ :

$$
\operatorname{Pr}(\mathcal{A}, \mathcal{B})=\min \left\{1, e^{-\frac{C(\mathcal{B})-C(\mathcal{A})}{T_{e}}}\right\}
$$

The cooling function decreases gradually the temperature parameter $T_{e}$ using the following equation: $T_{e}^{(\text {new })}=r * T_{e}^{(\text {old })}$, where $r$ is a predefined constant $(0<r<1)$.

The initial solution is a random allocation that can represent the state of a system at a given instance. Using the initial allocation, RAMA can be described as follows:

1. Determine an initial temperature $T_{e}$.

2. Find the Transit allocation $\mathcal{B}$ of $\mathcal{A}$ from $\mathcal{N}_{e}(\mathcal{A})$ and compute the transition probability $\operatorname{Pr}(\mathcal{A}, \mathcal{B})$.

3. If the cost function $C(\mathcal{B})$ is smaller than $C(\mathcal{A})$, then transit to allocation $\mathcal{B}$ with probability 1 . Otherwise, transit to the new allocation with probability $\operatorname{Pr}(\mathcal{A}, \mathcal{B})$.

4. If $\mathcal{B}$ is accepted, then go to step 5. Otherwise, repeat from step 2 using $\mathcal{A}$.

5. If $T_{e}<1$, then go to step 6 . Otherwise, reduce $T_{e}$ using the cooling function and return to step 2 using $\mathcal{B}$.

6. Halt after saving the best computed solution as an approximation of the optimal solution.

\section{Simulations And Results}

Using snapshot simulations and considering 5\% error margin, we evaluate the maximum capacity of the TD-CDMA/TDD system. More specifically, the maximum capacity is reached when less than $5 \%$ of mobiles in all snaphots have a CIR smaller than $\gamma_{t h}=-17 \mathrm{~dB}$. The error margin corresponds to the outage probability and reduces the sensitivity of the computed capacity to small differences in the performance-index.

\section{A. System Model}

We assume that the simulation area is finite, i.e. no wraparound is used. This implies that border effects will affect the results but that will be the case in a real UMTS TDD systems as well. We consider hexagonal micro-cells with $0.3 \mathrm{~km}$ radius. The assumed propagation model is a Xia-Bertoni model [16] with shadowing: $P_{r}=P_{e} \frac{k}{d_{x, y}^{\nu}} a_{x, y}$, where $P_{r}$ and $P_{e}$ are respectively the received and the transmitted powers, $k$ and $\nu$ are constants [17], which depend on the type of environment, and $d$ is the distance between the transmitter and the receiver. Factor $a_{x, y}$ models the shadowing effect as a time constant, lognormal variable with zero mean [18]. Transmitted power constraints and thermal noise power are inspired from [19]. We assume that a joint detection technique is used and that $\beta_{d}=0.1$. All simulation parameters are presented in table 1.

\section{B. Results}

We evaluate RAMA in a system with seven cells and seven slots using 120 different mobile geographic distributions. In figure 2, we present the Cumulative Distribution Function $(\mathrm{CDF})$ of the obtained $\gamma_{n}^{(s)}$ for a system with $10 \mathrm{mo}-$ biles/cell/slot. These are the final results that give the maximum capacity with RAMA when it is combined with WMM. As we can see, the probability of unsatisfied mobiles is less than 5\% when RAMA uses the WMM startegy. From the intermediate results (not shown here), the maximum capacity of the random allocation and RAMA when combined with BMM was estimated to 8 and 9 mobiles/cell/slot respectively. Hence, RAMA increases the capacity by $25 \%$ and $12.5 \%$ respectively when the WMM and BMM startegies are used. The results 
Table 1: Simulation parameters

\begin{tabular}{l|l}
$N_{0}$ & $-98 \mathrm{dBm}$ \\
Max BTS power & $33 \mathrm{dBm}$ \\
BTS PC range & $30 \mathrm{~dB}$ \\
$k$ & $-128.1 \mathrm{~dB}$ \\
$\nu$ & $37.6 \mathrm{~dB}$ \\
$\operatorname{std}\left(a_{x, y}\right)$ & $7 \mathrm{~dB}$ \\
$\operatorname{initial~} T_{e}$ & 100 \\
$r$ & 0.9
\end{tabular}

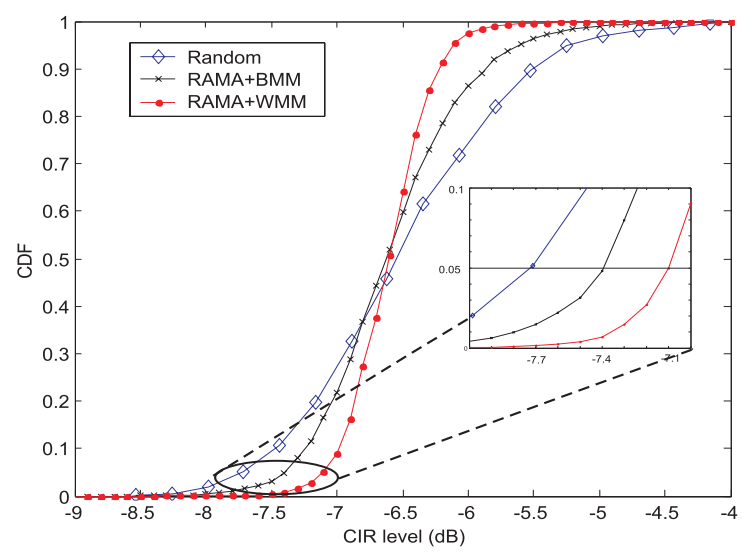

Figure 2: The CDF of the CIR in a system with 7 cells, 7 slots and $10 \mathrm{mobiles} / \mathrm{cell} / \mathrm{slot}$

show that the WMM is better than the BMM for capacity estimation due to the fast convegence of WMM and the fact that it consider the swap of the worst mobile. The latter can change drastically the maximum achieveble CIR in the allocated and released slots. We emphasize here that the number of iterations needed when WMM and BMM are used are respectively 12 and 43 iterations in average. It can be noticed that the number of allocations increases when using BMM strategy. This is due to the relatively slow convergence of this method. Finally, we note that the number of all possible allocations in this system is around $3 \times 10^{174}$. Thus, the complexity reduction is very important. From these results, we can deduce that the maximum capacity of a TD-CDMA/TDD system in downlink is around 10 mobiles/cell/slot. Thus, the maximum allowed capacity of 16 mobiles/cell/slot could not be achieved even under centralized quasi-optimal allocation and power control.

\section{SUMMARY}

In this paper, we have transformed the problem of computing the maximum capacity of a TD-CDMA/TDD system to a set of combinatorial optimization problems. This new formulation allows us to easily estimate system maximum capacity. Each intermediate-problem is solved by separating two independent optimization problems: optimum power control and slot allocation problems. The power control problem was solved using a simplified CIR-balancing mechanism while the slot allocation problem was solved using a proposed meta-heuristic algorithms based on simulated annealing. Simulation results have shown that the performance of RAMA is substantially high compared to the performance of the random allocation. However, we still unable to know how much the results of these algorithms are contiguous to the optimum solution. Hence, the development of new re-allocation algorithms using other metaheuristic is the objective of future works. In addition, future works involve the extension of these algorithms to uplink and crossed slots with different services using the simplified CIRbalancing power control. Finally, the new formulation could be extended to OFDMA where the slot allocation could be replaced by sub-carrier allocation.

\section{REFERENCES}

[1] W. Mohr, "Investigation of the UMTS radio interface in the ACTS FRAMES project," in Proc. of the UMTS workshop, IEEE ComSoc Germany, university of Ulm, vol. 1, 1998, pp. 33-64.

[2] W. S. Jeon and D. G. Jeong, "Comparison of time slot allocation strategies for cdma/tdd systems," IEEE Journal on Selected Areas in Communications, vol. 18, pp. 1271-1278, 2000.

[3] C. Y. Lee and K. W. Sung, "Dynamic resource allocation for CDMATDD indoor wireless systems," Wireless Communications and Mobile Computing, vol. 3, pp. 921-931, 2003.

[4] J. Nasreddine and X. Lagrange, "A simplified generic optimum power control scheme for CDMA cellular systems," in Proc. of the IEEE Workshop on Adaptive Wireless Networks, 2004, pp. 414-418.

[5] S. A. Grandhi, J. Zander, and R. Yates, "Constrained power control," Inter. Journal of Wireless Personal Communications, vol. 1, 1995.

[6] S. Kirkpatrick, C. D. J. Gellat, and M. P. Vecchi, "Optimization by simulated annealing," Science, vol. 220, pp. 671-680, 1983.

[7] "Physical channels and mapping of transport channels onto physical channels (TDD)," Third Generation Partnership Project, Technical Specification Group Radio Access Network, TS25.221.

[8] R. M. Karp, "Reducibility among combinatorial problems," in Proc. of a Symposium on the Complexity of Computer Computations, 1972, pp. 85-103.

[9] P. V. Hentenryck, M. Michel, and F. Benhamou, Newton: Constraint Programming over Nonlinear Constraints. Science of Computer Programming, 1997.

[10] M. R. Garey and D. S. Johnson, Computers and Intractability: A Guide to the Theory of NP-Completeness. W. H. Freeman and Co., 1979.

[11] S. Ammari and A. Wautier, "Interference factor evaluation for CDMA system capacity analysis," in Proc. of the IEEE Vehicular Technology Conference, vol. 3, 2001, pp. 1573 - 1577.

[12] S. A. Grandhi, R. Vijayan, D. J. Goodman, and J. Zander, "Centralized power control in cellular radio systems," IEEE Transactions on Vehicular Technology, vol. 42, pp. 66 - 468, 1993.

[13] R. S. Varga, Matrix iterative analysis. Prentice-Hall, 1962.

[14] J. Zander, "Performance of optimum transmitter power control in cellular radio systems," IEEE Transactions on Vehicular Technology, vol. 41, pp. $57-62,1992$.

[15] J. Nasreddine, Radio Resource Management in TD-CDMA/TDD Systems. Ph.D. thesis, University of Rennes 1, 2005.

[16] L. R. Maciel, H. L. Bertoni, and H. H. Xia, "Unified approach to prediction of propagation over buildings for all ranges of base station antenna height," IEEE Transactions on Vehicular Technology, vol. 42, pp. 41-45, 1993.

[17] AG-Siemens, "TDD UE-UE interference simulations," TSG-RAN Working Group 4 (Radio meeting n26), 2003.

[18] J. Nasreddine and X. Lagrange, "Time slot allocation based on path gain division scheme for TD-CDMA TDD systems," in Proc. of the IEEE Vehicular Technology Conference, 2003, pp. 1410-1414.

[19] "RF system scenarios," Third Generation Partnership Project,, Technical Specification Group Radio Access Network, TS25.942. 